\title{
Urologists should have a working knowledge of the evaluation and management of POP
}

\author{
Colin Birch, MD
}

Obstetrics and Pelvic Reconstructive Surgery, University of Calgary, Calgary, AB, Canada

Cite as: Can Urol Assoc J 2017;11 (6Suppl2):S131. http://dx.doi.org/10.5489/cuaj.4676

See related article on page $\mathrm{S} 125$.

$\mathrm{T}$ he pelvic floor has historically been the domain of the gynecologist. As we recognize the pelvic floor cannot be simply divided by anatomy and therefore by specialty, there is a move to abandon these divisions in favour of a multidisciplinary approach. Urologists who are involved in the care of female incontinence should be aware of the association of this condition with pelvic organ prolapse (POP). As such, in their evaluation of incontinence, they should have a working knowledge of the assessment and management of POP, thus allowing a comprehensive treatment plan to be established.

POP, like urinary incontinence, is a common problem, although its actual prevalence is unknown. Often this problem goes unreported, as POP often remains asymptomatic until there is significant pelvic organ descent. The pelvic floor is a dynamic system that can be damaged through childbirth and chronic increases in intra-abdominal pressure (chronic cough, constipation, and heavy work) leading to POP. As the Canadian population ages, this problem will require increased attention, as the "baby boomer" generation demands treatments that are current and effective.

This primer provided by Dr. Bureau and Dr. Carlson offers a review of the evaluation and management of POP that will provide the practicing urologist with a useful resource. The evaluation of POP relies on a combination of history and physical examination. In the history, it is important to not only enquire to the physical symptoms of the prolapse, but also the effects on urinary, bowel, and sexual function. The use of a standardized grading system (Pelvic Organ Prolapse Quantification [POP-Q]) allows not only the evaluation of the various compartments of the vagina, but can also be used to assess the outcomes of surgical treatments and as a communication tool between practitioners.

Management of POP should be individualized, discussing both conservative and surgical approaches. Initially, one should plan to correct underlying causes with weight loss, smoking cessation, and correction of constipation. Conservative measures, such as pelvic floor exercise (PFE) and pessary usage are often effective managements and should be offered as first-line treatments. The use of pessary has been viewed as an old management useful only for patients who are not fit for surgery. To the contrary, younger patients are increasingly using this option.

With failure of conservative measures or as a result of patient preference, surgical correction of POP is undertaken. As highlighted in this primer, surgical management of POP is an evolving field and requires accurate preoperative evaluation to choose the most appropriate surgical procedure. This is 'quality of life' surgery, which is not only intended to correct the anatomical defect, but also to maintain/enhance sexual, bowel, and bladder function. In even the most skilled hands, failure rates of $20 \%$ are expected, and this should be explained to patients considering surgical intervention. POP surgery has come under increased scrutiny from patients, hospitals, government agencies, and lawyers following the U.S. Food and Drug Administration safety warnings pertaining to the use of mesh products to augment native tissue repairs. The use of mesh inserted through a vaginal incision was introduced without adequate safety data, and long-term studies of effectivity did not confirm the expected durability of these repairs.

This primer recognizes that pelvic floor dysfunction is a common problem that can concurrently present as incontinence (urinary/fecal) and POP. The urologist managing female urinary incontinence should have a working knowledge of the evaluation and management of POP.

Competing interests: The author reports no competing personal or financial interests.

Correspondence: Dr. Colin Birch, Obstetrics and Pelvic Reconstructive Surgery, University of Calgary, Calgary, AB, Canada; Colin.Birch@albertahealthservices.ca 